Research Paper

\title{
Identification of Caveolin-I as a Potential Causative Factor in the Generation of Trastuzumab Resistance in Breast Cancer Cells
}

\author{
Sreeja C Sekhar ${ }^{1}$, Tomonari Kasai ${ }^{\boxplus}{ }^{\bowtie}$, Ayano Satoh ${ }^{1}$, Tsukasa Shigehiro ${ }^{1}$, Akifumi Mizutani1 ${ }^{1}$, Hiroshi Mu- \\ rakami ${ }^{1}$, Bishoy YA El-Aarag2 ${ }^{2}$ David S. Salomon ${ }^{3}$, Anna Massaguer ${ }^{4}$, Rafael de Llorens ${ }^{4}$, Masaharu Seno ${ }^{1}$ \\ 1. Division of Chemistry and Biotechnology, Graduate School of Natural Science and Technology, Okayama University, Okayama 7008530, \\ Japan. \\ 2. Department of Chemistry, Faculty of Science, Menofia University, Egypt. \\ 3. Mouse Cancer Genetics Program, Center for Cancer Research, National Cancer Institute, Frederick, Maryland 2702, USA. \\ 4. Biochemistry and Molecular Biology Unit, Department of Biology, Girona University, Girona 17071, Spain.
}

$\square$ Corresponding author: Tomonari Kasai. Telephone: +81-86-251-8265, Fax: +81-86-251-8216 E-mail: t-kasai@cc.okayama-u.ac.jp.

(c) Ivyspring International Publisher. This is an open-access article distributed under the terms of the Creative Commons License (http://creativecommons.org/ licenses/by-nc-nd/3.0/). Reproduction is permitted for personal, noncommercial use, provided that the article is in whole, unmodified, and properly cited.

Received: 2013.04.15; Accepted: 2013.06.17; Published: 2013.06.21

\begin{abstract}
The oncogenic tyrosine kinase receptor ErbB2 is a prognostic factor and target for breast cancer therapeutics. In contrast with the other ErbB receptors, ErbB2 is hardly internalized by ligand induced mechanisms, indicating a prevalent surface expression. Elevated levels of ErbB2 in tumor cells are associated with its defective endocytosis and down regulation. Here we show that caveolin-I expression in breast cancer derived SKBR-3 cells (SKBR-3/Cav-I) facilitates ligand induced ErbB2 endocytosis using an artificial peptide ligand EC-eGFP. Similarly, stimulation with humanized anti ErbB2 antibody Trastuzumab (Herceptin) was found to be internalized and co-localized with caveolin-I in SKBR-3/Cav-I cells. Internalized EC-eGFP and Trastuzumab in SKBR-3/Cav-I cells were then delivered via caveolae to the caveolin-I containing early endosomes. Consequently, attenuated Fc receptor mediated ADCC functions were observed when exposed to Trastuzumab and EC-Fc (EC-I peptide conjugated to Fc part of human IgG). On the other hand, this caveolae dependent endocytic synergy was not observed in parental SKBR-3 cells. Therefore, caveolin-I expression in breast cancer cells could be a predictive factor to estimate how cancer cells are likely to respond to Trastuzumab treatment.
\end{abstract}

Key words: ErbB2, Caveolin-1, Antibody dependent cell mediated cytotoxicity (ADCC), internalization, Ec-eGFP, Trastuzumab.

\section{Introduction}

As a co-receptor for other ErbB's, oncogenic receptor tyrosine kinase ErbB2, which lacks a natural ligand, can transduce strong mitogenic signals for cell transformation and proliferation by its ability to heterodimerize with the EGF receptor and with ErbB3 [1-3]. Correlation between the overexpression of ErbB2 and poor clinical prognosis is well documented in breast cancer patients, which marks this protein as a potential and effective therapeutic target [4, 5]. Controlled receptor activation and down regulation of receptor signaling are crucial for intracellular processes that are engaged by ligand binding. One of the ErbB2 targeting therapy is the administration of a humanized monoclonal antibody Trastuzumab, that binds to juxta membrane domain of ErbB2 [6, 7]. Clinical trials have shown that Trastuzumab inhibits 
breast cancer cell proliferation by the induction of cell cycle arrest at the G1 phase of the cell cycle [8]. Trastuzumab prevents ErbB2 hetero-dimerization with other ErbB receptors [9] and prevents its proteolytic shedding by metalloproteases [10]. Furthermore, the Fcy portion of Trastuzumab is reported to elicit antibody-dependent cell mediated cytotoxicity (ADCC) [11, 12], which probably is the dominant therapeutic activity of Trastuzumab, as Trastuzumab is ineffective in eliciting anti-tumor response in mice that lack Fcy receptors on ADCC promoting effector cells [13]. Taking the range of molecular and cellular mechanisms of Trastuzumab efficacy and of resistance into consideration, it might be possible that the dominant mechanism depends upon the context of the cancer target cell. However, in most cases, the levels of the ErbB2 surface expression on tumor cells are crucial for a potent and sustained therapeutic response to Trastuzumab.

Ligand induced endocytosis of receptor tyrosine kinases is considered to be crucial for the termination of intracellular signals that are transiently generated upon the ligand-receptor binding [14, 15]. Unlike ErbB1, which is a characterized receptor that undergoes endocytic processing and endosomal transport, the endocytosis of ErbB2 has been found to be impaired in tumor cells [16-19]. Moreover, several groups have reported that overexpression of cell surface ErbB2 can transmit its endocytic resistance to ErbB1 and its dimerization partners[17-19], resulting in a subsequent increase in the expression levels of the ErbB receptor heterodimerization partners on the cell membrane. Clathrin dependent endocytosis is a well-established phenomena among the ErbB family members [20-22]. However, the distribution of ErbB2 homo dimers in caveolae before ligand activation [23, 24 ] and its association with membrane proteins, lipid rafts, caveolins and flotillins are well documented [25-27]. These observations imply the involvement of caveolae-dependent mechanisms in ErbB2 endocytosis. Caveolin-1 is $22-24 \mathrm{kDa}$ integral membrane protein that is essential for caveolae formations, which are small, flask-shaped invaginations of the plasma membrane. In the context of endocytic function, caveolin-1 stabilizes the actin cytoskeleton and regulates the endocytic machinery by interacting with other membrane proteins [28-30]. Simultaneously, caveolae are often described as a signaling platforms, since caveolins bind to a number of signaling proteins including $G$ protein subunits, receptor and non receptor tyrosine kinases and small GTPases [31] thereby regulating their activity in caveolae. It has been demonstrated that overexpression of recombinant caveolin-1 regulates ErbB2 mediated signal transduction in vivo [32]. Although caveolin-1 has been reported to function as both a tumor suppressor and a oncogene, the cumulative effect on tumorgenesis may be dependent on tumor cell type. Deciphering the molecular mechanism(s) and physiological significances of ErbB2 endocytosis through a caveolin dependent-pathway, might well provide new insights for novel ErbB2 targeted therapies.

Until now, an endogenous ligand(s) for ErbB2 have not yet been identified. However, a phage display study has identified several small peptides that exhibit specific binding to the extracellular domain of ErbB2 [33]. These peptides inhibit the kinase activity of ErbB2 and subsequent cell proliferation. Previously, we described the EC-1 peptide, an artificial ErbB2 ligand, fused to eGFP (EC-eGFP) that efficiently induced ErbB2 endocytosis in the ovarian cancer cell derived SKOV-3, while EC-eGFP did not induce ErbB2 internalization in SKBR-3 cells [34]. Simultaneously, by DNA microarray analysis, we found that the mRNA level of caveolin-1 in SKBR-3 cells were down-regulated [35]. In this study, using Ec-eGFP and Trastuzumab, we evaluated the involvement of caveolin-1 in ligand induced ErbB2 endocytosis by utilizing parental SKBR-3 and SKBR-3/Cav-1 cells, in which the caveolin-1 gene was stably transduced.

\section{Materials and Methods}

\section{Antibodies and other reagents}

Rabbit monoclonal anti-ErbB2, anti-Caveolin-1, anti-Caveolin-2, mouse monoclonal anti-Transferrin antibody, HRP conjugated anti-mouse IgG, anti-rabbit IgG, anti-rabbit IgG labeled Alexa Fluor 350 were from Cell Signaling Technology. Anti-EEA1 and anti- $\beta$-actin antibodies were purchased from BD Biosciences. Trastuzumab (Herceptin) was purchased from (Genentech). Anti-rabbit IgG labeled Alexa Fluor 555 and 488, anti-mouse IgG labeled Alexa Flour 555 was from Invitrogen. Anti-human IgG FITC was from Sigma. Protein Assay reagents were from Thermo Scientific, VA. All other reagents used in this study were purchased from Sigma-Aldrich and Invitrogen unless otherwise specified.

\section{Cell cultures and Transfection of Caveolin-I into SKBR-3 cells}

The human breast cancer derived cell line SKBR-3 and the human ovarian cancer cell line SKOV-3 (ATCC, Manassas, VA) were grown in supplemented RPMI 1640 medium at $37^{\circ} \mathrm{C}$ with $10 \%$ FBS, under an atmosphere of $5 \% \mathrm{CO}_{2}$. Full length human caveolin-1 cDNA derived from SKOV-3 cells were amplified using forward primer 5'-gcggecgcatgtct 
gggggcaaatac-3' and reverse primer $5^{\prime}$-gaattcttatatttc tttctacaagttg-3'. RT-PCR products were digested with Not1 and EcoR1 and then ligated to the Not1/EcoR1-digested PQCXIP retrovirus vector (Clontech, Mountain View, CA) to generate PQCXIP/Cav1. The GP2-293 cell line (Clontech) was used to produce the viral particles. Production of virus particles, infection of the target cell line SKBR-3 and selection of virus infected SKBR-3 cells were performed as recommended by the vendor. Cells were transfected in parallel with the PQCXIP vector as a control. Puromycin $(0.8 \mu \mathrm{g} / \mathrm{l})$ resistant stable caveolin-1 expressing transfectants SKBR-3 cells were generated and verified using anti -caveolin- 1 antibody (1:500).

\section{Preparation of Ec-eGFP and EC-FC}

EC-eGFP and EC-Fc (EC-1 peptides fused to human IgG Fc domain) proteins were prepared as previously described [34, 35]. Briefly, for EC-eGFP, synthetic oligo nucleotide coding for EC-1 peptide, $\mathrm{NH}_{2}$-WTGWCLNPEESTWGFCTGSF-COOH, and eGFP coding DNA were cloned into an expression vector pET28b (Novagen, WI, USA). A Full length EC-eGFP expressing plasmid was then introduced into E. coli MM294 (DE3) pLysS cells. For purification, cells harboring pET28b were grown at $37^{\circ} \mathrm{C}$ in LB media, induced protein expression at mid-exponential phase by adding $0.4 \mathrm{mM}$ IPTG, grown for an additional $16 \mathrm{~h}$, harvested and sonicated by resuspending in cold PBS. After centrifugations for $15 \mathrm{~min}$ at $25,000 \times g$, the supernatant was collected and applied to a Ni-NTA super flow column (Qiagen) pre-equilibrated with $50 \mathrm{mM}$ phosphate buffer $\mathrm{pH} 8.0$ and $300 \mathrm{mM} \mathrm{NaCl}$. EC-eGFP protein was eluted with $300 \mathrm{mM}$ imidazole and was dialyzed against PBS for 2 $h$ and the purity of recombinant EC-eGFP protein was analyzed by SDS-PAGE.

To generate EC-Fc, the above expression vectors [35] were stably transfected into Chinese hamster ovary (CHO) cells in the presence of $100 \mu \mathrm{g} / \mathrm{ml}$ hygromycin B (Wako Pure-chemicals, Japan). Six million of the transformed cells were seeded in $500 \mathrm{ml}$ of CHO-S-SFM II (Invitrogen) at $37^{\circ} \mathrm{C}$ and incubated for 5 days. The cell culture medium was collected on the 5th day, centrifuged, and passed through a pre-equilibrated protein A Sepharose CL-4B column. After extensive washes, the bound protein was eluted in $1 \mathrm{ml}$ fractions in $0.1 \mathrm{M}$ phosphate buffer of $\mathrm{pH}$ 2.6, and was immediately neutralized by adding $20 \mu \mathrm{l}$ of 2 $\mathrm{M}$ phosphate buffer $\mathrm{pH}$ 8.0. The eluted EC-Fc proteins were then passed through a PD10 column (GE Healthcare) to remove the buffer and resuspended in PBS.

\section{siRNA Transfection}

siRNAs to cav-1 (5'-aagcatcaacttgcagaaaga-3') and cav-2 (5'-ccggctcaactcgcatctcaa-3') (QIAGEN) were transiently transfected into SKOV-3 cells using Lipofectamine LTX (Invitrogen) to knockdown the gene expression of caveolin-1 and caveolin-2 according to the manufacturer's instructions.

\section{Cell Surface Biotinylation}

Cell surface biotinylation assay, using cleavable biotin was used to determine the amount of internalized ErbB2 in transfected and wild type SKBR-3 cells. Briefly, 80-90\% confluent SKBR-3 cells cultured in 6 well plates were washed twice with Hank's balanced salt solution (HBSS) for $10 \mathrm{~min}$ at $4^{\circ} \mathrm{C}$. EZ-Link ${ }^{\mathrm{TM}}$ Sulfo-NHS-SS-Biotin (Pierce, Thermo Scientific, Rockford, IL) at a concentration of $0.5 \mathrm{mg} / \mathrm{ml}$ in HBSS was added to cells and placed on a shaking table for $20 \mathrm{~min}$ at $4^{\circ} \mathrm{C}$. This reaction was repeated twice. Biotinylation was quenched by washing the cells with HEPES buffered RPMI supplemented with 1\% BSA and $2 \mathrm{mM}$ glutamine for $10 \mathrm{~min}$ at $4^{\circ} \mathrm{C}$. Control cells prepared for $37^{\circ} \mathrm{C}$ and $4^{\circ} \mathrm{C}$ were incubated with RPMI-BSA for $1 \mathrm{~h}$. SKBR-3 cells incubated with 30 $\mu \mathrm{g} / \mathrm{ml}$ Ec-eGFP and $1 \mu \mathrm{M}$ Trastuzumab were kept at $37^{\circ} \mathrm{C}$ for different time intervals. Fresh cold RPMI-BSA was added to the cells upon completion of each time point (except for the controls), and the cells were then placed on ice. A similar set of samples processed at $4^{\circ} \mathrm{C}$ were also kept as controls. After incubation, all the samples were washed once in cold HBSS and non-internalized cell surface biotin was removed by washing twice in stripping buffer $(20 \mathrm{mM}$ DTT; $50 \mathrm{mM}$ Tris-HCl pH 8.7; $100 \mathrm{mM} \mathrm{NaCl}$ and 2.5 $\mathrm{mM} \mathrm{CaCl} 2$ ) for $20 \mathrm{~min}$ at $4^{\circ} \mathrm{C}$. Finally, cells were washed three times in cold HBSS, and lysed in RIPA buffer (50 mM Tris- $\mathrm{HCl} \mathrm{pH} 7.4 ; 150 \mathrm{mM} \mathrm{NaCl} ; 0.1 \%$ sodium dodecyl sulphate (SDS); $1 \%$ Triton X-100; $0.5 \%$ sodium deoxycholate; $1 \mathrm{mM}$ EDTA, Protease and Phosphatase inhibitor cocktail (Sigma-Aldrich), incubated for $20 \mathrm{~min}$ at $4^{\circ} \mathrm{C}$ and sonicated twice. Cell extracts were cleared by centrifugation for $5 \mathrm{~min}$ at $25,000 \times \mathrm{g}$ and supernatants were incubated with $20 \mu \mathrm{l}$ of avidin-agarose (Pierce Chemical) at $4^{\circ} \mathrm{C}$ overnight. Beads were washed three times in RIPA buffer, suspended in Laemmlli buffer $(20 \%$ glycerol, $135 \mathrm{mM}$ Tris- $\mathrm{HCl} \mathrm{pH}$ 6.8, 4\% SDS, 10\% 2-mercaptoethanol, $0.005 \% \mathrm{BPB})$, heated for $5 \mathrm{~min}$ at $95^{\circ} \mathrm{C}$ to release the bounded proteins and processed for western blotting.

\section{Western blotting}

Protein samples lysed in Laemmlli sample buffer and were then boiled for $5 \mathrm{~min}$. Equal quantities of protein lysates were then analyzed on SDS-PAGE and 
transferred to PVDF membrane (Bio-Rad, Hercules, CA). The membranes were blocked with $10 \%$ skim milk, incubated with primary antibody in $0.4 \%$ skim milk/TBS for $2 \mathrm{~h}$, washed with TBST followed by appropriate secondary antibody in $0.4 \%$ skim milk/TBS incubation for $1 \mathrm{~h}$. The proteins were visualized using Western lighting plus-ECL reagent (PerkinElmer) in Light-Capture II cooled CCD camera system (ATTO, Tokyo, Japan).

\section{Immunofluorescence analysis and confocal microscopy}

For confocal microscopic observation, SKBR-3 cells and caveolin-1 transfected SKBR-3 cells were grown on $18 \mathrm{~mm}$ cover slips. Cells were then fixed with $4 \%$ PFA for $10 \mathrm{~min}$, permeabilized with $0.1 \%$ Triton X-100/PBS and blocked with 1\% BSA-PBS for $30 \mathrm{~min}$ at RT. For caveolin-1 localization, cells were incubated with 1:500 diluted anti-caveolin-1 antibody in $0.25 \%$ BSA/PBS for $1 \mathrm{~h}$ at RT, followed by staining with Alexa 555 labeled anti-rabbit IgG for $30 \mathrm{~min}$. After wash, the cover slips were mounted with DAPI (Vector Laboratories and observed by confocal microscope IX81 with 60x magnification lens. (Olympus, Tokyo, Japan).

\section{Analysis of internalized ErbB2}

To analyze the internalized ErbB2 that was potentially co-localized with caveolin-1, cells were incubated with $30 \mu \mathrm{g} / \mathrm{ml}$ Ec-eGFP or $1 \mu \mathrm{g} / \mathrm{ml}$ Trastuzumab in $1 \%$ BSA PBS at $4^{\circ} \mathrm{C}$ (data not shown) and $37^{\circ} \mathrm{C}$. After incubation, the cells were fixed with $4 \%$ PFA for $10 \mathrm{~min}$, permeabilized with $0.1 \%$ Triton X-100/PBS and blocked with 1\% BSA-PBS for $30 \mathrm{~min}$ at RT. Cells were incubated with primary antibodies: anti-rabbit Caveolin-1, ErbB2 or anti- mouse EEA1 diluted in 1:500 with BSA/PBS for $1 \mathrm{~h}$ at $37^{\circ} \mathrm{C}$ followed by appropriate secondary antibody staining for 30 min at RT. Cells were imaged as described earlier.

\section{Lactate Dehydrogenase release for ADCC as- say}

Caveolin-1 expressing and wild type SKBR-3 and SKOV-3 cells that had been cultured in 96 well plates were incubated with $1 \mu \mathrm{M}$ of EC-Fc and Trastuzumab for $4 \mathrm{~h}$ at $37^{\circ} \mathrm{C}$. Peripheral blood monocytes (PBMC) were isolated from human whole blood sample that were obtained from a healthy donor using Ficoll plus (Invitrogen) gradient centrifugation. Isolated PBMCs were washed three times with PBS and incubated together with antibody pre-treated SKBR-3 and SKOV-3 cells at an effector: target ratio of 25:1, 50:1,100:1 for $4 \mathrm{~h}$ at $37^{\circ} \mathrm{C}$. After $4 \mathrm{~h}, 96$ well plates were centrifuged at $1,300 \times \mathrm{g}$ for $10 \mathrm{~min}$. The cell superna- tant was then transferred to a fresh 96 well plate to determine the amount of LDH released using colorimetric assay (LDH Assay Kit, Promega) as per the vendor's protocol. The percentage of cytotoxicity was calculated as: (experimental - effector spontaneous target spontaneous) / (target maximum - target spontaneous) $x 100$, where the "experimental" corresponds to the signal measured in a treated sample, "effector spontaneous" corresponds to the signal measured in the presence of PBMCs alone, "target spontaneous" corresponds to the signal measured in the presence of tumor cells alone, and "target maximum" corresponds to the signal measured in presence of 1\% TritonX-100 lysed cells.

\section{Statistical Analysis}

The Data is expressed as the mean $\pm \mathrm{SE}$. The statistical significance of differences between means was determined using Student's $t$ test. Differences were statistically significant at $P<0.05$.

\section{RESULTS}

\section{Localization of ectopic caveolin-I in SKBR-3 cells}

Previous work by us [34, 35] and others [30] indicated the loss of caveolin-1 gene expression and caveolae in SKBR-3 cells. In order to investigate the relevance to ErbB2 internalization, a construct constitutively expressing human full-length caveolin-1 was transfected into SKBR-3 cells. Immunofluorescence microscopy of transfected cells demonstrated caveolin-1 was frequently expressed on the cell membrane (Fig. 1A), and also as small or large punctae vesicles throughout the cytoplasm (Supplementary Material: Fig. S2A), which may represent caveosomes, identified previously by Pelkmans et al. [37]. The cellular localization of these vesicles were similar to the localization pattern as reported previously in SKBR-3 [30], and that of SKOV3 (Supplementary Material: Fig. S2B), which endogenously expresses caveolin-1 [34]. Cell lysates prepared from transfected and wild type SKBR-3 cells were subjected to western blot analysis with caveolin-1 specific monoclonal antibody (Fig.1B). A significant increase in caveolin-1 protein levels was detected in transfected cells whereas wild type SKBR-3 cells were caveolin-1 negative, consistent with our previous study [34]. SKOV-3 cells were used as positive controls for analysis since caveolin-1 was detected in SKOV-3 [34]. The results confirmed the expression of recombinant caveolin-1 in the transfected SKBR-3 cells (SKBR-3/Cav-1), which was equivalent with the level of endogenous caveolin-1 in SKOV-3 cells. 
A
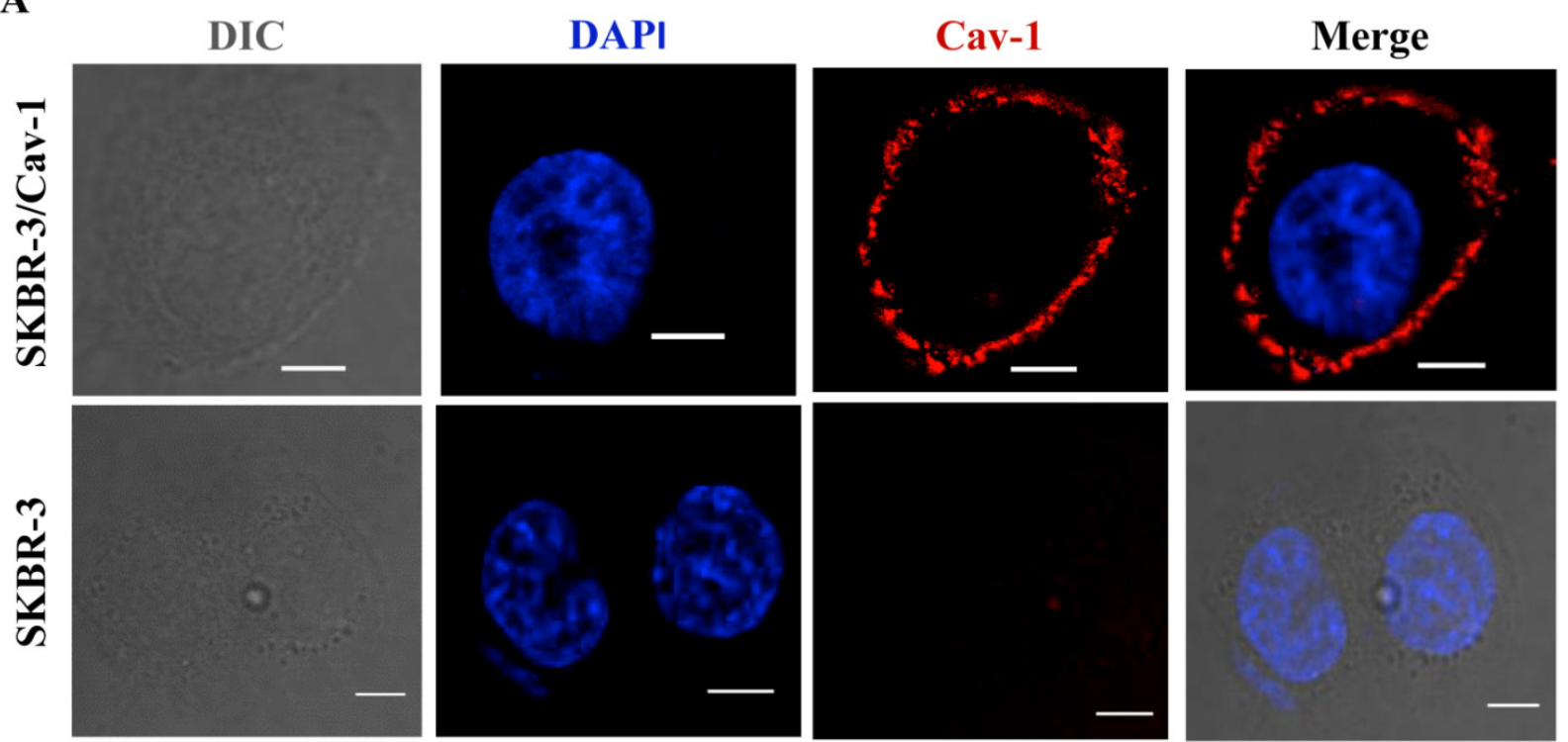

B

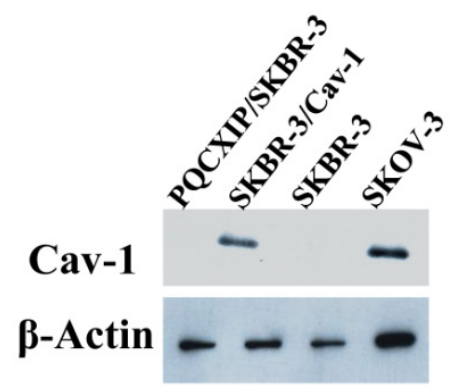

Fig I. Expression of caveolin-I in SKBR-3 cells. (A) Both SKBR-3/Cav-I and parental SKBR-3 cells were processed for immunofluorescence imaging, detecting bright field image in (DIC) caveolin-I (red) and nuclei (blue). Scale bar: $10 \mu \mathrm{m}$. (B) Western blot showing caveolin-I expression in, PQCXIP vector transfected (lane I), Caveolin-I transfected (SKBR-3/Cav-I) (lane 2) parental SKBR-3 (lane 3), and SKOV-3 cells (lane 4). $\beta$-Actin was used as loading control.

\section{ErbB2 internalization is enhanced in SKBR-3 cells expressing caveolin-I}

To address whether efficient ErbB2 endocytosis is associated with caveolin-1 expression in SKBR-3 cells, we treated caveolin-1 transfected SKBR-3 cells and wild type cells with EC-eGFP, an artificial ErbB2 peptide ligand. Cell surface binding of EC-eGFP were observed together with ErbB2 within 5 min of incubation (Fig. 2A). However, induced endocytosis and intracellular localization of EC-eGFP was observed in transfected cells after $15 \mathrm{~min}$ of incubation at $37^{\circ} \mathrm{C}$ (Fig. 2B). In wild type SKBR-3 cells, ErbB2 was retained on the cell surface even after $60 \mathrm{~min}$ of incubation with EC-eGFP at $37^{\circ} \mathrm{C}$ (Fig.2 C). We also analyzed the effect of Trastuzumab in caveolin-1 expressing SKBR-3 cells after ligand stimulation (Fig. 2D), and after $15 \mathrm{~min}$ of incubation at $37^{\circ} \mathrm{C}$. After the addition of EC-eGFP, the intracellular localization of ErbB2 in
SKBR-3/Cav-1 expressing cells was found to be abundant (Fig. 2E) whereas Trastuzumab above scarcely altered the cellular membrane distribution of ErbB2 in wild type SKBR-3 cells. Supportingly, siRNA directed silencing of caveolin-1 or caveolin-2 in SKOV-3 resulted in a reduction of EC-eGFP uptake (Supplementary Material: Fig. S1). These results suggest that upon the binding to ErbB2, EC-eGFP and Trastuzumab are effectively internalized with ErbB2 through a caveolin-1 dependent mechanism. As a negative control for endocytosis, we incubated SKBR-3/Cav-1 cells with eGFP and analyzed the endocytosis and localization of ErbB2 and caveolin-1. Cell surface binding of GFP was not observed whereas naked ErbB2 and caveolin-1 were localized on the cell membrane (Supplementary Material: Fig.S3). 

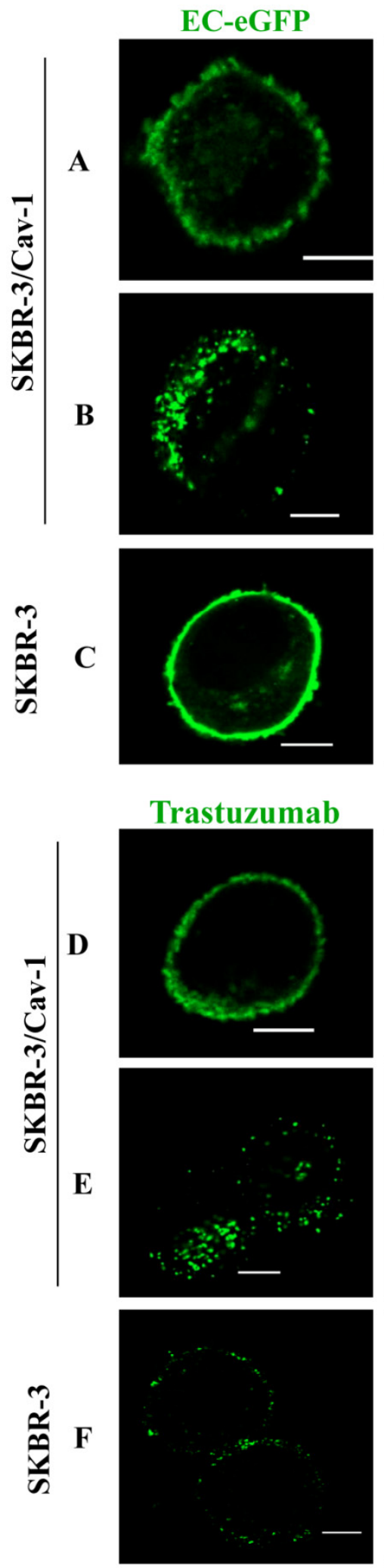
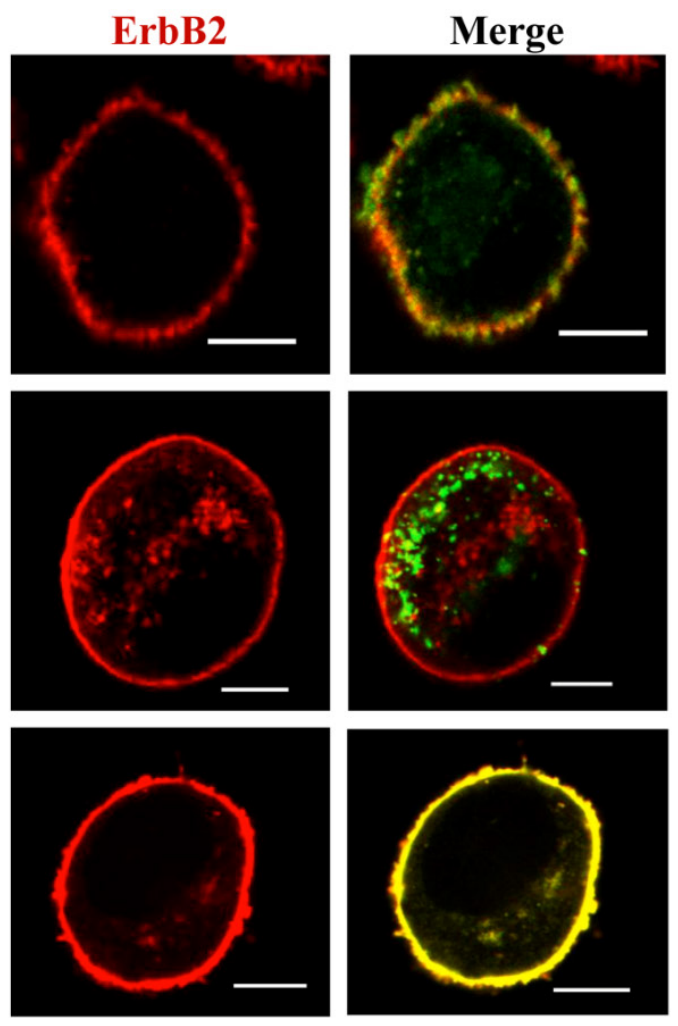

Cav-1
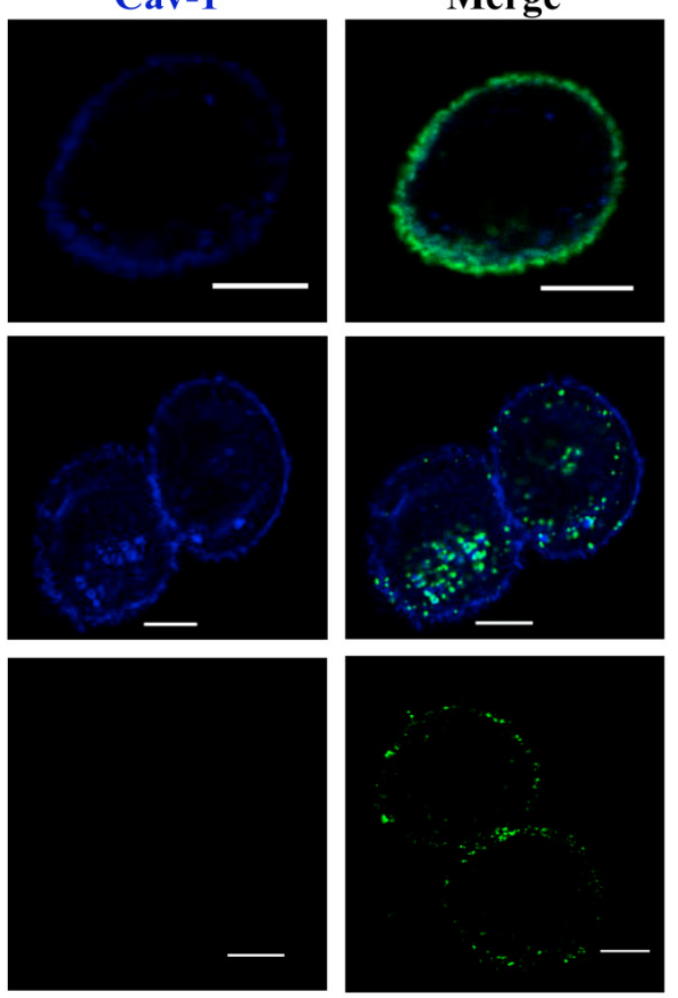

Fig 2. Enhanced internalization of ErbB2 in caveolin-I expressing SKBR-3 cells. (A) SKBR-3/Cav-I cells were incubated with I $\mu M$ of EC-eGFP at $37^{\circ} \mathrm{C}$ for 5 min and internalization was assessed using anti-ErbB2 monoclonal antibody. Cell surface binding of EC-eGFP (green) with ErbB2 (red) was shown in the merged picture (yellow). (B) enhanced endocytosis of Ec-eGFP (green) and Intracellular co-localization with ErbB2 (red) was observed after 15 min of incubation at $37^{\circ} \mathrm{C}$. (C) Parental SKBR-3 cells incubated with EC-eGFP for $15 \mathrm{~min}$ at $37^{\circ} \mathrm{C}$ were processed for endocytosis analysis as above using anti ErbB2 antibody (red). (D) SKBR-3/Cav-I cells incubated with Trastuzumab (green) for $5 \mathrm{~min}$ at $37^{\circ} \mathrm{C}$ were processed and stained with anti-caveolin-I antibody (blue); cell surface binding of Trastuzumab with caveolin-I was shown (opaque) in the merged image. (E) Internalization and localization of Trastuzumab (green) with caveolin-I (blue) in SKBR-3/Cav-I cells after I 5 min of stimulation at $37^{\circ} \mathrm{C}$. (F) Parental SKBR-3 cells incubated similarly with Trastuzumab for $15 \mathrm{~min}$ were processed for endocytosis analysis as detailed in the material and methods section. Scale bar: $20 \mu \mathrm{m}$. 
We further accessed for the involvement of caveolin-1 in ligand induced ErbB2 endocytosis by biotinylation (Fig.3). Surface biotinylated cells were treated with $1 \mu \mathrm{M}$ EC-eGFP and Trastuzumab at $37^{\circ} \mathrm{C}$ for various intervals. Surface biotinylated proteins on cell surface were then removed to ensure only internalized biotinylate proteins were being assessed. Cell lysates were pull-downed with avidin agarose and blotted against anti-ErbB2 monoclonal antibody to analyze the endocytosed ErbB2 in both caveolin-1 transfected and wild type SKBR-3 cells. As for the control, the same set of samples, but the ligand or Trastuzumab incubation were carried out at $4^{\circ} \mathrm{C}$, were processed simultaneously for internalization. Internalization of the transferrin receptor was also monitored as an endocytosis control. In EC-eGFP treated caveolin-1 transfectants, the amount of ErbB2 which was pulled down with avidin-beads was increased 15-30 min after ligand treatment (Fig. 3A). This was followed by a gradual decrease within $90 \mathrm{~min}$, presumably due to potential endosomal/lysosomal degradation. In contrast, no significantly pulled down of ErbB2 was detected in caveolin-1 transfected SKBR-3 cells which had been incubated with ligand at $4^{\circ} \mathrm{C}$ and in wild type SKBR-3 cells after EC-eGFP treatment (Fig. 3B). Trastuzumab was also found to increase the amount of biotinylated ErbB2 gradually with time in the cytoplasm of caveolin-1 transfected SKBR-3 cells, and partially in wild type SKBR-3 cells at $60 \mathrm{~min}$ (Fig. 3B). Since only internalized proteins were biotinylated and could be pulled down with avidin-beads under our experimental condition, we concluded that ErbB2 was endocytosed upon the ligand/Trastuzumab binding through a caveolin-1 mediated pathway.

$\mathbf{A}$
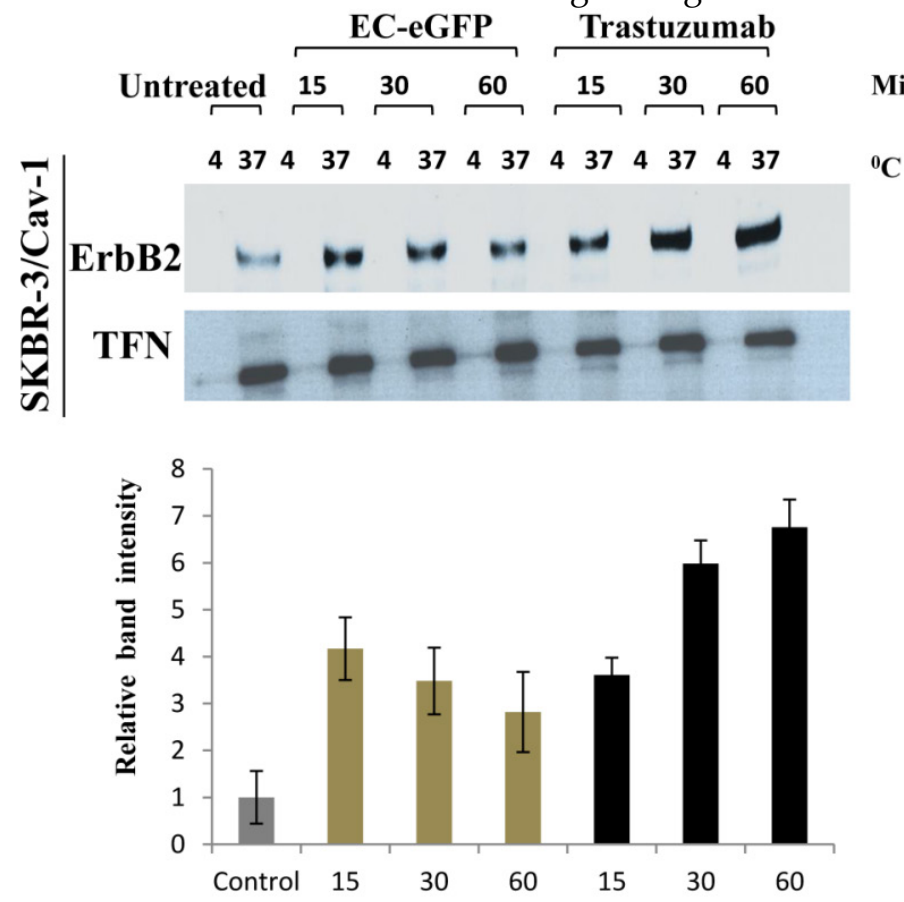

B
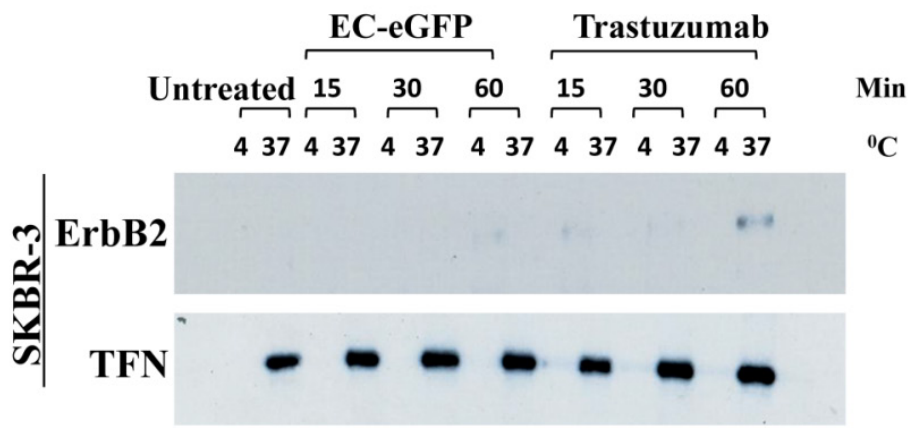

Fig 3. Ligand induced ErbB2 internalization after EC-eGFP and Trastuzumab treatment. SKBR-3 cells were surface biotinylated to monitor internalization. (A) Biotinylated caveolin-I expressing SKBR-3 cells were stimulated with I $\mu$ M EC-eGFP and Trastuzumab for I5, 30 and 60 min at $37^{\circ} \mathrm{C}$, $4^{\circ} \mathrm{C}$ or left untreated for I h at $37^{\circ} \mathrm{C}$ and $4^{\circ} \mathrm{C}$. Densitometry was performed using Image J software. (B) Wild type SKBR-3 cells were incubated with Ec-eGFP and Trastuzumab as described above for 15-60 min. Cell lysates prepared from treated samples were then pull-downed with avidin agarose and subjected to western blotting using an anti-ErbB2 antibody. Endocytosed transferrin receptor was also monitored simultaneously as internal control. The results are expressed as the mean SD of three individual experiments. Since there was no significant internalization observed in parental SKBR-3 cells, densitometry was performed only for caveolin-I expressing SKBR-3 cells. 
Moreover, studies with endosomal markers to monitor post-endocytic events revealed the fusion of caveolin-1 rich vesicles with early endosomal compartments [36]. We thus analyzed the recruitment of EC-eGFP and Trastuzumab into early endosomes using an early endosomal marker EEA1 after EC-eGFP and Trastuzumab stimulation in caveolin-1 transfected SKBR-3 cells. Localization in endosomes was not observed in transfected cells after $1 \mu \mathrm{M}$ EC-eGFP or Trastuzumab treatment for initial $15 \mathrm{~min}$ at $37^{\circ} \mathrm{C}$ (data not shown). However, enhanced endocytosis and localization in early endosomes was observed in transfected cells after EC-eGFP treatment for $90 \mathrm{~min}$ at $37^{\circ} \mathrm{C}$ (Fig. 4A). Trastuzumab also found to be localized in early endosomes after incubation for $1 \mathrm{~h}$ (Fig. 4B). Co-localization of caveolin-1 with EEA1 was observed beneath the cell membrane, and as small and large caveolin-1 positive endosomal vesicles which were similar to those reported in previous studies [37, 38]. In line with those observations, these results imply a post-endocytic intersection of a caveolin-1 mediated pathway with the classic endosomal systems.

\section{Attenuated ADCC effect on SKBR-3 cells ex- pressing caveolin- I}

To assess if the level of ErbB2 endocytosis is sig- nificant for SKBR-3 cells to escape from the ADCC effect, we set out to evaluate the ADCC effect mediated by human peripheral blood mononuclear cells (PBMC) on Trastuzumab or EC-FC treated caveolin-1 transfected SKBR-3 and SKOV-3 cells (Fig. 5). The PBMC mediated cytotoxicity of wild type of SKBR-3 cells was taken as controls for the ADCC effect. When the effector/target cell ratio was 100:1, 50:1, 25:1, cell death was approximately 70,55 and $45 \%$, respectively in the case of wild type of SKBR-3. In contrast, a significant attenuated ADCC cell killing effect was observed in caveolin-1 expressing SKBR-3 cells with a $20-35 \%$ cell death, which was almost equivalent to the ADCC effect observed in SKOV-3 cells. This was in agreement with the protein levels of caveolin-1 in both cells types (Fig. 1B). The trend of ADCC effects were similarly observed with both Trastuzumab and EC-Fc, suggesting that antibody or ligand induced internalization of ErbB2 through a potential caveolin-1 dependent pathway could be significant for desensitizing cells to the ADCC-dependent cellular cytotoxicity of Trastuzumab.

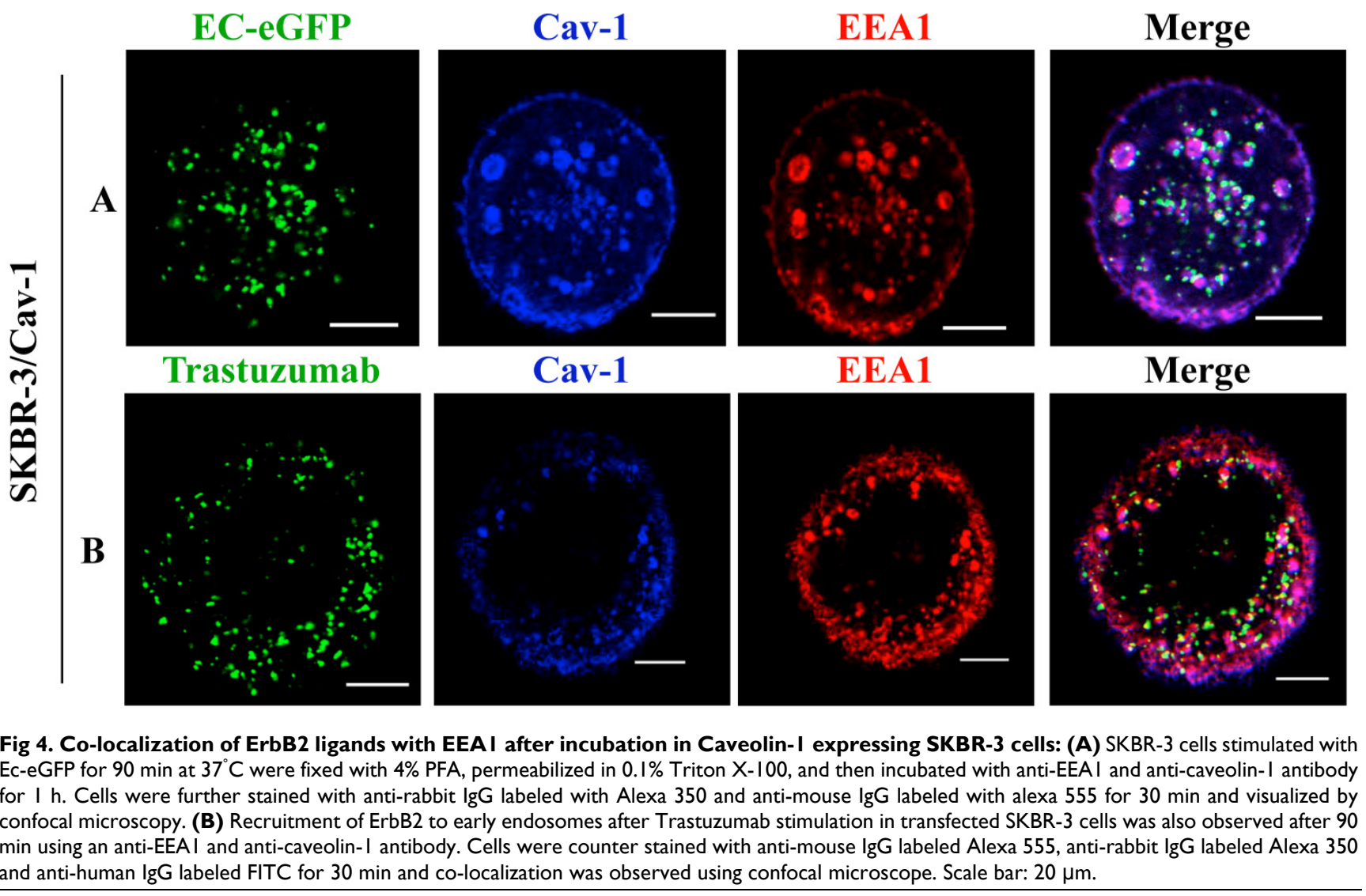


A
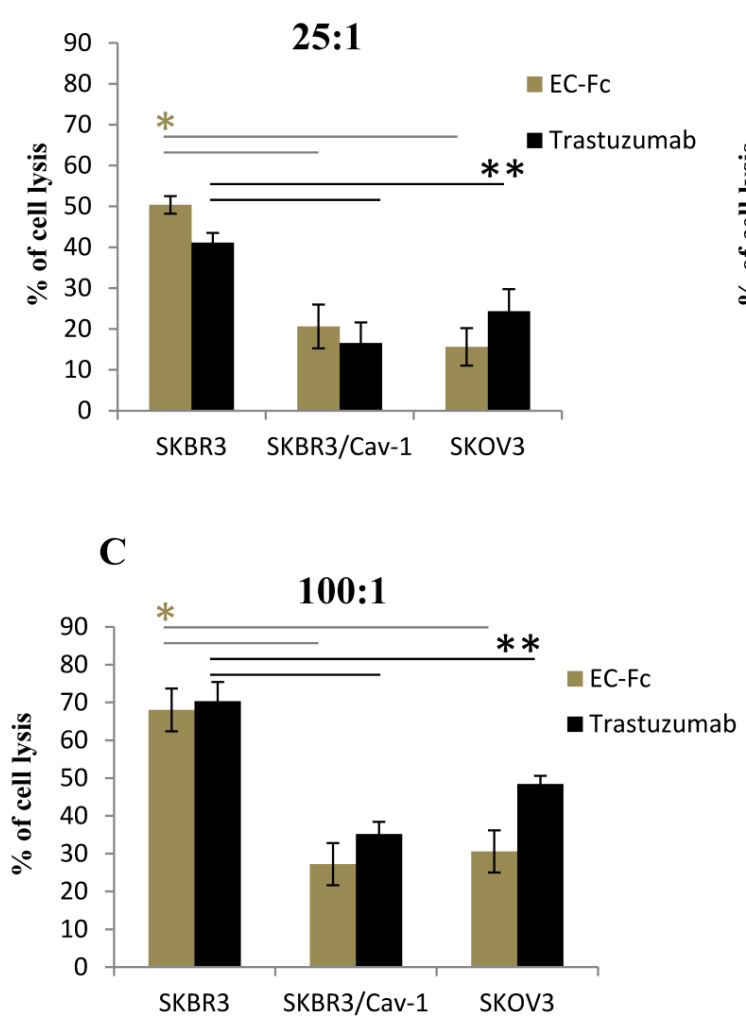

B

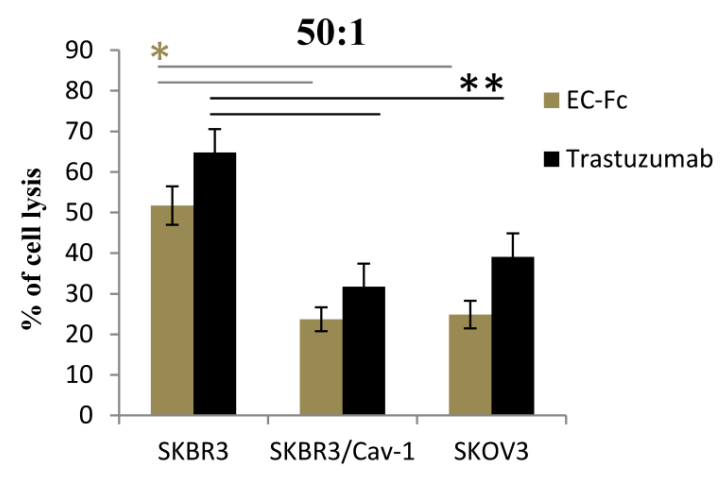

Fig 5. Fc receptor mediated ADCC in SKBR-3 cells. ADCC activity mediated by Trastuzumab and EC-Fc against cell surface ErbB2 in SKBR-3, SKBR-3/Cav-I and SKOV-3 cells was measured using human PBMC's as effector cells at an effector: target cell ratio of (A) 25:I (B) 50:I and (C) I00:I, with standard LDH assay as described in materials and methods. Data are expressed as the mean of $( \pm)$ SD ( $n=3)$. Student's t- test (two tailed) was used to compare the ADCC response in SKBR-3/Cav-I and SKOV-3 with parental SKBR-3. Differences were statistically significant at $P<0.05$.

\section{DISCUSSION}

This is the first report to describe a positive correlation between ligand induced ErbB2 endocytosis and caveolin-1 expression in SKBR-3 cells. Upon EC-eGFP or Trastuzumab treatment, ErbB2 was internalized in caveolin-1 expressing SKBR-3 cells (Fig. 2) and in SKOV-3 cells that express endogenous caveolin-1 [34]. Reciprocally, the inhibition of ErbB2 internalization by siRNA mediated depletion of caveolin-1 and 2 in SKOV-3 cells resulted in a decreased internalization of ErbB2, which further confirmed the involvement of caveolin-1 in mediating endocytosis (Supplementary Material: Fig. S2). In this context, caveolae may well function as an endocytotic pathway for ErbB2 internalization. On the other hand, significant differences in the ADCC response in caveolin-1 transfected and wild type SKBR-3 cells mediated by Trastuzumab and EC-Fc further substantiates a possible functional effect of ErbB2 endocytosis in Trastuzumab resistance.

The EC-1 peptide and Trastuzumab might directly crosslink cell-surface ErbB2 thereby favoring clustering of a peptide- or antibody-receptor complex to form homo-dimers. Elevated homo dimerizations of ErbB2 after Trastuzumab stimulation in SKBR-3 cells was previously reported to localize in raft-associated regions of the cell membranes [23, 24, 38]. Caveolae/ caveolin structure and functions have also been shown to be raft associated [40]. In addition, flotillins, a raft-associated protein, stabilizes caveolin- 1 on the membrane [25], and facilitates complex formations with ErbB2 and Hsp90 [25]. Geldanamycin, a potential Hsp90 inhibitor, disrupts this complex and triggers ErbB2 endocytosis in SKBR-3 [41, 42]. It should be indicated here that those observations were performed in wild type SKBR-3 cell. However, our RT-PCR analysis revealed low amount of endogenous caveolin-1 in SKBR-3 [35], which might have physiological consequences in terms of internalization defects in SKBR-3 cells. Over expression of caveolin-1 should induce de novo formation of caveolae and therefore confer stability to caveolar vesicles [43]. Collectively with our findings, it can be speculated that in the presence of caveolin-1, the homo dimerizations of ErbB2 formed after Ec-eGFP/Trastuzumab 
treatment could facilitates membrane bound ErbB2 to internalize into caveolin-1 containing early endosomes which would develop into caveolae. Following internalization, the endocytosed molecules may well destined to the intracellular compartments for sorting and degradation. Internalized ErbB2 has been reported to co-localize with early endosomal markers after prolonged ligand stimulation [36]. This is in agreement with our observation in transfected SKBR-3 cells, where we observed the co-localization of ErbB2 with endosomal marker EEA1 after 90 min of Ec-eGFP/Trastuzumab treatment (Fig.4), implying a possible interaction between caveolin-1 mediated endocytosis with classic endosomal system through Rab5-GTPase. [37, 38]

Although the relationship between caveolin-1 and tumorogenesis has been debated [44], in breast cancer derived cells, the tumor suppressor efficacy of caveolin-1 may related to a receptor down regulation. It should be noted that a inverse relationship between caveolin-1 and ErbB2 expression has been reported in breast cancer [45-47]. Taking our results into consideration that ligand-stimulated ErbB2 facilitates endocytic uptake via caveolae, thus caveolin-1 should contribute to inhibit growth/proliferation signals from ErbB2, thereby functioning as a tumor suppressor $[48,49]$. On the other hand, caveolin- 1 and caveolae deficiency might be preferable in the clinical administration of Trastuzumab. As a consequence of enhanced endocytosis, we observed attenuation in $\mathrm{Fc}_{\mathrm{c}}$ receptor PBMC activity mediated by Trastuzumab and EC- Fc in caveolin-1 expressing SKBR-3 cells and SKOV-3 cells. An effector / target ratio dependent ADCC response was observed markedly in wild type SKBR-3 cells after 4 hrs of incubation with EC-FC /Trastuzumab (Fig. 5). ErbB2 was retained on the cell surface even after ligand stimulation in caveolae deficient breast cancer cells. The attenuated ADCC effect caused by the antibody mediated endocytosis of ErbB2 by caveolin-1 might be one mechanism that could contribute to Trastuzumab resistance. Thus, collectively, we propose caveolin-1 expressed SKBR-3 cells as another model to explain Trastuzumab resistance in ErbB2 overexpressing cell lines. The expression levels of caveolin-1 might be a predictive marker to distinguish tumor cells that respond favorably to Trastuzumab from Trastuzumab resistant ones.

\section{Supplementary Material}

Fig.S1 - Fig.S3.

http://www.jcancer.org/v04p0391s1.pdf

\section{Acknowledgements}

We thank Dr. Yoshiaki Iwasaki, M.D, PhD, Okayama University for his assistance to conduct ADCC experiments. This work was supported by JSPS KAKENHI Grant Numbers 24510151, 23650598.

\section{Conflict of interest}

The authors declare no conflict of interest.

\section{References}

1. Lemmon MA, Schlessinger J. Cell signalling by receptor tyrosine kinases. Cell. 2010; 141: 1117-1134

2. Sorkin A, Zastrow MV. Endocytosis and signalling: intertwining molecular networks. Nature reviews Molecular Cell Biology. 2009; 10: 609- 622

3. Hubbard R, Miller WT. Receptor tyrosine kinases: mechanisms of activation and signalling. Current Opinion in Cell Biology. 2007; 19: 117-123.

4. Bennasroune A, Gardin A, Aunis D, et al. Tyrosine kinase receptors as attractive targets of cancer therapy. Critical Reviews in Oncology/Hematology. 2004; 50: 23-38.

5. Baselga J, Swain SM. Novel anticancer targets: revisiting ERBB2 and discovering ERBB3. Nature Reviews Cancer. 2009; 9: 463-475.

6. Hudziak R M, Lewis GD, Winget M, et al. p185HER2 monoclonal antibody has anti proliferative effects in vitro and sensitizes human breast tumor cells to tumor necrosis factor. Molecular Cell Biology. 1989; 9: 1165-1172.

7. Carter P, Presta L, Gorman CM, Ridgway JB, et al. Humanization of an anti-p185HER2 antibody for human cancer therapy. Proc. Natl. Acad. Sci. 1992; 89: 4285-4289.

8. Lane H A, Beuvink I, Motoyama A B, et al. ErbB2 Potentiates Breast Tumor Proliferation through Modulation of p27 Kip1-Cdk2 Complex Formation: Receptor Over expression Does Not Determine Growth Dependency. Molecular Cell Biology. 2000; 20: 3210- 3223

9. Junttila TT, Akita RW, Parsons K, et al. Ligand-independent HER2/HER3/PI3K complex is disrupted by trastuzumab and is effectively inhibited by the PI3K inhibitor GDC-0941. Cancer Cell. 2009; 15: 429-440.

10. Molina MA, Codony-servat J, Albanell J, et al. Trastuzumab (Herceptin), a Humanized Anti-HER2 Receptor Monoclonal Antibody, Inhibits Basal and Activated HER2 Ectodomain Cleavage in Breast Cancer Cells. Cancer Research. 2001; 61: 4744 -4749.

11. Peipp M, Dechant M, Valerius T. Effector mechanisms of therapeutic antibodies against ErbB receptors. Current Opinion in Immunology. 2008; 20: 436-443.

12. Arnould L, Gelly M, Penault-Llorca F, et al. Trastuzumab-based treatment of HER2-positive breast cancer: an antibody-dependent cellular cytotoxicity mechanism? British Journal of Cancer. 2006; 94: 259- 67.

13. Clynes RA, Towers TL, Presta LG, Ravetch JV. Inhibitory Fc receptors modulate in vivo cytotoxicity against tumor targets. Nature Medicine. 2000; 6: 443-6.

14. Birtwistle MR, Hatakeyama M, Yumoto N B, et al. Ligand-dependent responses of the ErbB signaling network: experimental and modeling analyses. Molecular Systems Biology. 2007; 3: 144.

15. Sorkin A, Goh LK. Endocytosis and intracellular trafficking of ErbBs. Experimental Cell Research. 2009; 315: 683- 696.

16. Baulida J, Kraus MH, Alimandi M, et al. All ErbB receptors other than the epidermal growth factor receptor are endocytosis impaired. The Journal of Biological Chemistry. 1996; 271: 5251-7.

17. Roepstorff K, Grøvdal L, Grandal M, et al. Endocytic downregulation of ErbB receptors: mechanisms and relevance in cancer. Histochemistry and Cell Biology. 2008; 129: 563-578.

18. Shen F, Lin $Q$, Childress $C$, Yang W. Identification of the domain in ErbB2 that restricts ligand-induced degradation. Cellular Signalling. 2008; 20: 779- 86 .

19. Hommelgaard AM, Lerdrup M, Deurs BV. Association with Membrane Protrusions Makes ErbB2 an Internalization-resistant Receptor. Molecular Biology of the Cell. 2004; 15: 1557-1567.

20. Haslekås C, Breen K, Pedersen KW, et al. The Inhibitory Effect of ErbB2 on Epidermal Growth Factor-induced Formation of Clathrin-coated Pits Correlates with Retention of Epidermal Growth Factor Receptor-ErbB2 Oligomeric Complexes at the Plasma Membrane. Molecular Biology of the Cell. 2005; 16: 5832-5842. 
21. Sigismund S, Woelk T, Puri C, et al. Clathrin-independent endocytosis of ubiquitinated cargos. Proc. Natl. Acad. Sci. 2005; 102: 2760-2765.

22. Schlessinger J. Dimerization and activation of EGF receptor. Cell. 2002; 110: 669-672.

23. Nagy P, Vereb G, Sebestyén Z, et al. Lipid rafts and the local density of ErbB proteins influence the biological role of homo- and heteroassociations of ErbB2. Journal of Cell Science. 2002; 115: 4251-4262.

24. Nagy P, Claus J, Jovin TM, Arndt Jovin DJ. Distribution of resting and ligand-bound ErbB1 and ErbB2 receptor tyrosine kinases in living cells using number and brightness analysis. Proc. Natl. Acad. Sci. 2010; 107: 16524-16529.

25. Kirkham M, Parton RG. Clathrin-independent endocytosis: New insights into caveolae and non-caveolar lipid raft carriers. Biochimica et Biophysica Acta (BBA)-Biomembranes. 2005; 1746: 350-363.

26. Pust S, Klokk TI, Musa N, et al. Flotillins as regulators of ErbB2 levels in breast cancer. Oncogene. 2012;: 1-9.

27. Austin CD, Mazie AM, Pisacane PI, et al. Endocytosis and Sorting of ErbB2 and the Site of Action of Cancer Therapeutics Trastuzumab and Geldanamycin. Molecular Biology of the Cell. 2004; 15: 5268-5282.

28. Hommelgaard AM, Roepstorff K, Vilhardt F. Caveolae: stable membrane domains with a potential for internalization. Traffic. 2005; 6: 720-4.

29. Römer W, Pontani LL, Sorre B, et al. Actin dynamics drive membrane reorganization and scission in clathrin-independent endocytosis. Cell. 2010; 140: 540-53.

30. Verma P, Ostermeyer-fay AG, Brown DA. Caveolin-1 Induces Formation of Membrane Tubules That Sense Actomyosin Tension and Are Inhibited by Polymerase I and Transcript Release Factor/Cavin-1. Molecular Biology of Cell. 2010; 21: 2226-2240.

31. Quest AFG, Gutierrez-Pajares J, Torres VA. Caveolin-1: an ambiguous partner in cell signalling and cancer. Journal of Cellular and Molecular Medicine. 2008; 12: 1130-50.

32. Engelman JA, Lee RJ, Karnezis A, et al. Reciprocal Regulation of Neu Tyrosine Kinase Activity and Caveolin-1 Protein Expression in Vitro and in Vivo. The Journal of Biological Chemistry. 1998; 273: 20448-20455.

33. Pero SC, Shukla GS, Armstrong AL, et al. Identification of a small peptide that inhibits the phosphorylation of ErbB2 and proliferation of ErbB2 overexpressing breast cancer cells. International Journal of Cancer. 2004; 111: 951-60.

34. Hashizume T, Fukuda T, Nagaoka T, et al. Cell type dependent endocytic internalization of ErbB2 with an artificial peptide ligand that binds to ErbB2. Cell Biology International. 2008; 32: 814 -26.

35. Vaidyanath A, Hashizume T, Nagaoka T, et al. Enhanced internalization of ErbB2 in SK-BR-3 cells with multivalent forms of an artificial ligand. Journal of Cellular and Molecular Medicine. 2011; 15: 2525-38.

36. Barr DJ, Ostermeyer-Fay AG, Matundan RA, Brown DA. Clathrin-independent endocytosis of ErbB2 in geldanamycin-treated human breast cancer cells. Journal of Cell Science. 2008; 121: 3155-66.

37. Pelkmans L, Bürli T, Zerial M, Helenius A. Caveolin-stabilized membrane domains as multifunctional transport and sorting devices in endocytic membrane traffic. Cell. 2004; 118: 767-780.

38. Liebl D, Difato F, et al. Mouse Polyomavirus Enters Early Endosomes, Requires Their Acidic $\mathrm{pH}$ for Productive Infection, and Meets Transferrin Cargo in Rab11-Positive Endosomes. Journal of Virology. 2006; 80: 4610-4622.

39. De LC, Cozzolino R, et al. Biological properties of a human compact anti-ErbB2 antibody. Carcinogenesis. 2005; 26: 1890- 1895.

40. Nabi IR, Le PU. Caveolae/raft-dependent endocytosis. The Journal of Cell Biology. 2003; 161: 673-7.

41. $\mathrm{Xu} \mathrm{W,} \mathrm{Mimnaugh} \mathrm{E,} \mathrm{Rosser} \mathrm{MF,} \mathrm{et} \mathrm{al.} \mathrm{Sensitivity} \mathrm{of} \mathrm{mature} \mathrm{ErbB2} \mathrm{to}$ geldanamycin is conferred by its kinase domain and is mediated by the chaperone protein Hsp90. The Journal of Biological Chemistry. 2001; 276: 3702-8.

42. Pedersen NM, Madshus IH, Haslekås C, Stang E. Geldanamycin-induced down-regulation of ErbB2 from the plasma membrane is clathrin dependent but proteasomal activity independent. Molecular Cancer Research. 2008; 6: 1-500.

43. Williams TM, Lisanti MP. The caveolin proteins. Genome Biology. 2004; 3: $1-8$.

44. Hino M, Doihara H, Kobayashi K, et al. Caveolin-1 as tumor suppressor gene in breast cancer. Surgery Today. 2003; 33: 486-90.

45. Lee SW, Reimer CL, Phil Oh, et al. Tumor cell growth inhibition by caveolin re-expression in human breast cancer cells. Oncogene.1998; 16: 1391-1397.

46. Sagara Y, Mimori K, Yoshinaga K, et al. Clinical significance of Caveolin-1, Caveolin-2 and HER2/neu mRNA expression in human breast cancer. British journal of Cancer. 2004; 91: 959-965.
47. Park SS, Kim JE, Kim YA, et al. Caveolin-1 is down-regulated and inversely correlated with HER2 and EGFR expression status in invasive ductal carcinoma of the breast. Histopathology. 2005; 47: 625-630.

48. Patani N, Martin LA, Reis-Filho JS, Dowsett M. The role of caveolin-1 in human breast cancer. Breast Cancer Research and Treatment. 2012; 131: $1-15$.

49. Sloan E K, Ciocca DR, Pouliot N, et al. Stromal cell expression of caveolin-1 predicts outcome in breast cancer. The American Journal of Pathology. 2009; 174: 2035-43. 